\title{
Lifecycle Optimization of Smart Contract for Flexibility
}

\author{
Hong Su
}

\begin{abstract}
Smart contract-based methods are used to implement the blockchain applications. While smart contracts have separate pre-deployed steps, which are suitable for applications that are deployed once and invoked many times. However, there are smart contracts that are used only one time (the disposable smart contract) or few times. Pre-deployment requires an additional step and additional transactions, which bring burdens (such as longer waiting time and more transaction fees) to users. In this paper, we propose a new Lifecycle model of smart contracts, which allows combining the pre-deployment with function invocations. This facilitates the usage of the disposable smart contract, as users are only required to send one transaction to perform both the pre-deployment and the function invocation. Together with the smart contract separation, it also allows participants to customize their special smart contracts at the request time. At last, we verify the proposed model and it shows the potential to save additional burden and to facilitate the usage of the smart contract.
\end{abstract}

Index Terms—smart contract, pre-deployment, lifecycle optimization, flexibility.

\section{INTRODUCTION}

$S_{\text {in }}^{\text {sind }}$ MART contracts are software on the blockchain, which intend to automatically execute actions or rules. They are often used to implement different blockchain applications. For example, smart contracts are used in electricity or energy trading [1] [2], IP Circuit Protection [3].

Before using a smart contract, there is a separate predeployment step, which puts the smart contract to the blockchain and instantiates it. This step costs extra money and users have to wait additional time before real functional invocations. It is not a big burden for the applications that are invoked many times later, as the cost of the deployment is relatively small for each invocation.

However, there are disposable smart contracts. It is a kind of smart contract that is invoked only once. They are for specific scenarios, such as for specific peers or specific time. An example is a contract between two traders. It is always different, as the contract changes for different time, different goods, or other specific negotiation contents. A predeployment brings relative heaven burdens to this kind of smart contracts, as one deployment is only for one function invocation.

The pre-deployment process has different kinds of limitations, and we summarize them as follows.

(1) The pre-deployment step requires additional transactions, which results in two aspects: (a) Users have to wait for several blocks to make sure that the pre-deployment transaction has been put to the blockchain. Users cannot send its function invocations during the waiting time; otherwise, there may be an error condition that the invocation transaction is sealed into the blockchain while the smart contract is not. (b) To make those transactions put to the blockchain, senders have to pay rewards to the miner, which

- H. Su is with the College of Computer Science, Sichuan University, Chengdu, China, 610041. costs money. The cost can be significant if the smart contract is invoked few times.

(2) The smart contract is fixed after the pre-deployment. Change of a smart contract requires a new contract [4]. A user cannot change its smart contract even before its action is involved. (a) Participants cannot decide their requirements previously. Instead, they decide their requirement when they send out transactions to interact with the smart contract(s). In the trade example, participants may decide their trading terms and conditions when sending their transactions. (b) Different participants may have different requirements, which makes it difficult to have a unified smart contract.

It is ideal if the pre-deployment can be along with the function invocation. When there are no separate steps to deploy a smart contract, it helps to overcome the above limitations, especially if a smart contract is invoked few times.

In this paper, we propose to optimize the lifecycle steps of a smart contract. In this model, the first invocation can be along with the code deployment process. Meanwhile, for flexibility and compatibility, users can choose to whether combine those steps or not. The contributions of this paper are as follows.

(1) We propose a lifecycle optimization model, in which the deployment of a smart contract is along with its functional invocation. This model helps to save the predeployment transaction, and then it saves the corresponding transaction fee and the waiting time.

(2) We propose to use the lifecycle optimization model to delay users' specific smart contracts to the time when users send their requests to the smart contract. This is different from the current way that needs to predefine the requirements of all participants in a pre-deployed smart contract.

(3) We propose a new address format for the optimization of the lifecycle. This address identifies different seg- 
ments in a transaction. It consists of two parts, the address of the sending transaction, and a specific identifier. With this address, we can use one transaction to contain the information for the different lifecycle steps.

The rest of this paper is as follows. Section II describes the lifecycle optimization model, which allows combining different lifecycle steps. Section III describes and analyzes the different characteristics of the lifecycle model. Section IV shows the verification results, and section $\mathrm{V}$ describes related works. Section VI concludes this paper.

\section{LIFECYCLE OPTIMIZATION}

This describes the lifecycle optimization model, with the aim to use a smart contract flexibly. The flexibility of a smart contract means (1) it has fewer steps for a user to use a smart contract, and (2) it allows users to add smart contracts for new participants.

\subsection{Lifecycle Optimization Model}

We divide the lifecycle of a smart contract on a blockchain into several steps. The first step is to put the code of a smart contract into the blockchain. This step is called the codeputting step, abbreviated as ' $c$ ' or step ' $c$ '. The second step is to instantiate the smart contract, the instantiation step (abbreviated as ' $i$ ' or step ' $i$ '). In step ' $i$ ', the smart contract is loaded into the memory and an associated process (or instance) is created on blockchain nodes. Normally, a corresponding handles or address is associated with this instance. The third step is to invoke the corresponding interface of the smart contract by the user. This step is the invocation step, abbreviated as ' $\mathrm{v}$ ' or step ' $\mathrm{v}$ '. The predeployment process often contains step ' $c$ ', and ' $i$ '. There is a termination step, which we discuss separately in the subsection of "Termination Optimization" for simplification.

Fig. 1. The proposed model. Users can select to run ' $c$ ', 'i', and ' $v$ ' separately or in the combined way.

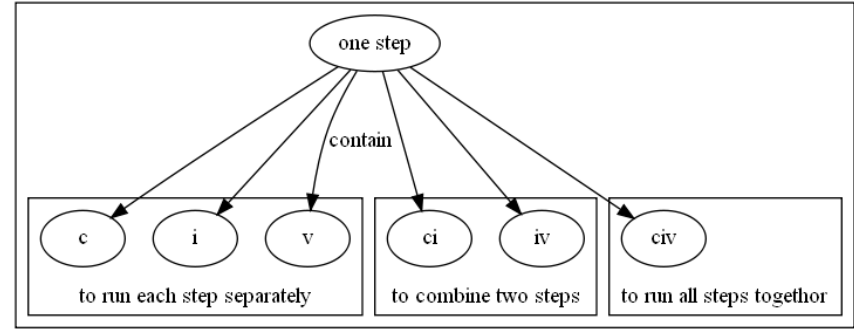

Based on the above lifecycle steps, we propose a new model, in which the lifecycle steps are combined into one step. A user can put the code of a smart contract to the blockchain, instantiate the smart contract, and invoke its interface together. Meanwhile, this model also allows users to select some of those steps to run at one time. This model is called the lifecycle optimization model. Figure 1 shows this model.

With this new model, a user has several selections for the lifecycle of a smart contract. For clarification, we list the possible cases that a user can do in one step.
TABLE 1

Possible Combinations of Different Smart Contract Lifecycle Steps

\begin{tabular}{|c|c|}
\hline combinations & meaning \\
\hline ci-v & $\begin{array}{l}\text { one transaction triggers step ' } \mathrm{c}^{\prime} \text { and ' } \mathrm{i} \text { ', another } \\
\text { transaction triggers step ' } \mathrm{v} \text { '. }\end{array}$ \\
\hline c-iv & $\begin{array}{l}\text { one transaction triggers step ' } c \text { ', another trans- } \\
\text { action triggers step ' } \mathrm{i} \text { ' and step ' } \mathrm{v} \text { '. }\end{array}$ \\
\hline civ & one transaction triggers step ' $c$ ', ' $i$ ', and step ' $v$ '. \\
\hline
\end{tabular}

(1) A sender can perform the above steps separately. In each step, the sender sends out a corresponding transaction to trigger it.

(2) A sender can do ' $c$ ' and ' $i$ ', or ' $i$ ' and ' $v$ ' in one step. In the former one, code is put into the blockchain and instantiate it. In the latter case, it instantiates the code and invokes the interface. In the latter case, the code should be put to the code into the blockchain previously or the instantiation will fail.

(3) A sender can do ' $c$ ', ' $i$ ', and ' $v$ ' together. One transaction does all the things. In this method, the code is putting to the blockchain, instantiated, and its interface is invoked.

To give a clear comparison of those combinations, we list the combination in Table 1.

Notice, if a user combines step of ' $c$ ' and step of ' $i$ ' into one step and does step ' $\mathrm{v}$ ' separately, it is the currently used model (to deploy a smart contract first and invoke its interface later).

To support the proposed model, we add new fields in the transaction data structure. Those fields contain three parts (or segments), which are corresponding to the lifecycle steps of a smart contract.

Fig. 2. The transaction data structure used for smart contract.

\begin{tabular}{|c|c|}
\hline \multicolumn{2}{|c|}{ transaction for smart contract } \\
\hline \multirow{3}{*}{$\begin{array}{l}\text { smart } \\
\text { contract }\end{array}$} & smart contract code \\
\hline & instantiation \\
\hline & invocation \\
\hline & $\ldots$ \\
\hline
\end{tabular}

The first part is the smart contract code segment. If this field exists, it indicates that the sender wants to put the smart contract code to the blockchain.

The instantiation segment contains the parameters to instantiate a smart contract. If this field exists, a smart contract instance is created. If several instances are required, the sender puts all instance parameters here one by one.

The invocation segment contains the parameters to invoke the interface of an instance. If this field exists, the according interface will be invoked.

\subsection{Address}

As both the smart contract code component and the instantiation component may be in one transaction, they are identified by different names (also called identifiers). Then their addresses are identifiers plus the transaction's address. 
Fig. 3. New address format.

\begin{tabular}{|l|l|}
\hline \multicolumn{2}{|c|}{ address of code or instance } \\
\hline identifier & address of transaction \\
\hline
\end{tabular}

With the new address mechanism, there are two possible ways to find a target smart contract or its instance. If the target is in the same transaction, the user can only use the identifier to find it. Algorithm 1 is an example, in which there are two smart contracts $\left(i d \_m\right.$, and $\left.i d \_n\right)$ in the same transaction $t x$. The invocation at line 11 uses the identifier $i d \_m$ to specify the target smart contract.

If the invocation is not in the same transaction, the target transaction is located first, and then the corresponding code or instance is found. Algorithm 2 is such an example; there are two smart contracts $\left(i d \_m\right.$ and $\left.i d \_n\right)$ in two transactions $(t x 1$, and $t x 2)$. The invocation at line 13 uses the identifier $i d \_m$ plus the address of $t x 1$ to specify the target smart contract.

Note that there may be several smart contracts in the code field. Identifiers can also be used to differentiate different smart contracts. For example, a smart contract may have its common library; the library needs an identifier to be used by other smart contracts. Similarly, in the instance field, there may also be several instantiation configurations. Each one stands for a separate instance and has its own identifier.

The address of instance is not required in the civ method if there is only one invocation. In this case, the invocation is inside its transaction, and thus the address of the instance is not used. The user does not care about the issue of address, which facilitates the user to use the disposable smart contract. It is different from the current way to have an instance address previously and then all invocations are through this address.

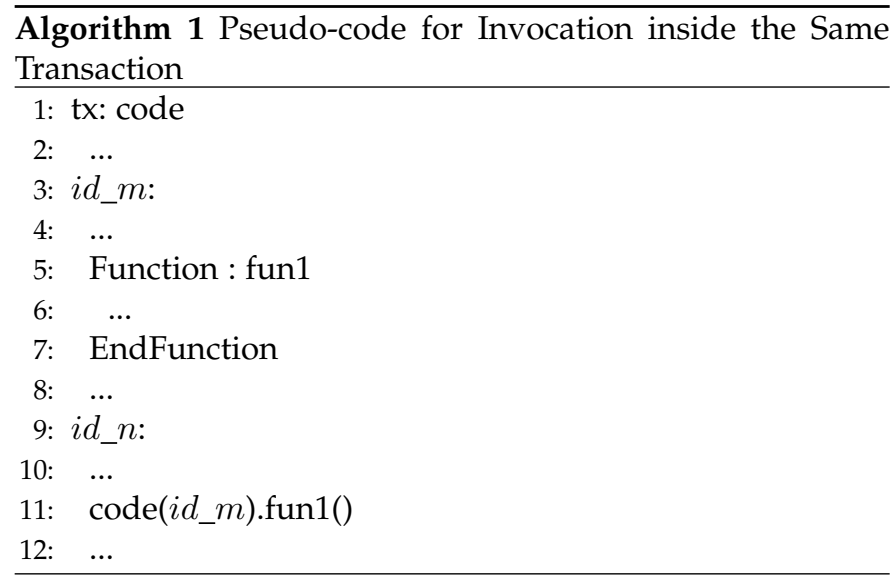

\subsubsection{Code Reuse}

Although all lifecycle actions are performed in the civ method, the code can still be reused. Other smart contracts can reuse the smart contract by its address.

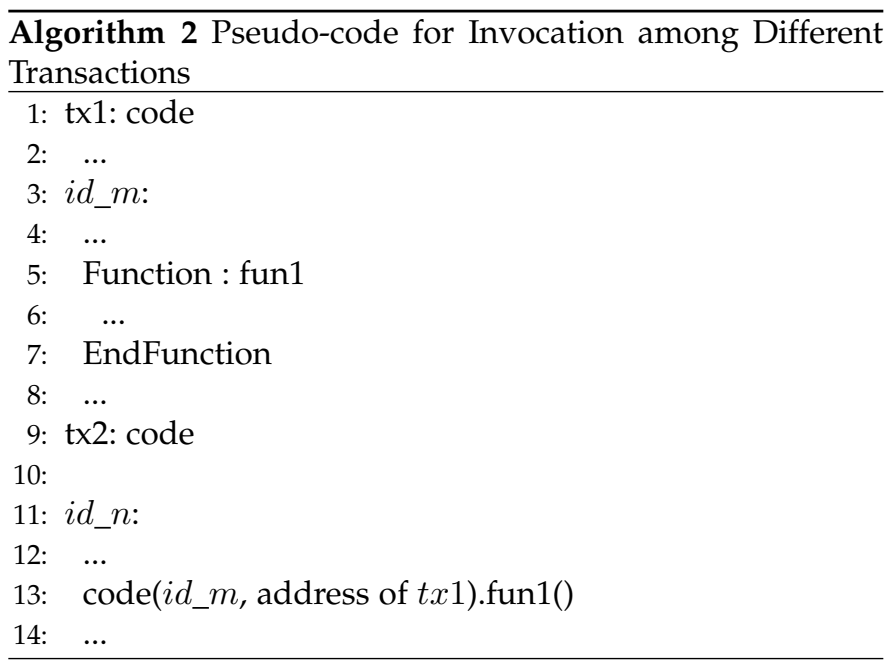

On the other hand, the smart contract by the civ method can invoke functions of other smart contracts. As it is disposable, it is often not a common logic; instead, it is a smart contract for a specific scenario, which changes often and should invoke codes of other smart contracts. It is more like the script language that invokes other OS commands and combines those commands.

If a smart contract contains both common logic and changeable logic, it can be divided into two parts and each part have an identifier. One is the common part, which is reused by other smart contracts; another part is the changeable part. Those two parts can still be done in one step.

\subsection{Termination Optimization}

The above discussion does not contain the termination step for simplification, and we discuss it separately here. If we consider this step, the possible combinations are civt, civ-t, ci-vt, ci-v-t, c-ivt, c-iv-t, c-i-vt, and c-i-v-t.

When this step is combined with other steps (such as civt, ci-vt, c-ivt, c-i-vt), it also saves one additional transaction. Especially, if a disposable smart contract is used only once, all lifecycle steps (' $c$ ', ' $i$ ', ' $v$ ', and ' $t$ ') can be combined in one step (civt). It saves two transactions than the currently used method (ci-v-t) (one transaction is used for the predeployment and another one is for the termination).

\section{ANALYSIS}

In this section, we analyze important characteristics of the proposed model, higher transaction utilization rate, possibility of dynamical logic, and enhanced robust. At last, we describe the scope of the optimization.

\subsection{Transaction Utilization Rate}

Transactions are divided into two types concerning their effect on a smart contract. One kind of transaction is used to invoke functions of a smart contract (used in step ' $\mathrm{v}$ '), called functional transactions. This is the aim of the users to use a smart contract. While another kind of transaction is used for the other lifecycle steps (such as step ' $c$ ', ' $i$ '), and those transactions are called non-functional transactions. 
It is helpful if we know the proportion of functional transactions. This measurement is called the transaction utilization rate (tur), and it is defined in (1). The higher a tur is, the more transactions are used to trigger functions of the smart contract.

$$
t u r=n \_f u n / a
$$

, where $n \_f u n$ is the number of functional transactions, and $a$ is the total number of transactions, which is the summarization of $n \_f u n$ and $n \_n o n f$ (the number of non-functional transactions).

$$
a=n \_f u n+n \_n o n f
$$

With tur, we can compare the transaction utilization ratio among different lifecycle methods. tur of civ, ci-v, and c-i-v methods are shown in (3).

$$
\begin{aligned}
& \operatorname{tur}_{c i v}=1 / 1=100 \% \\
& \operatorname{tur}_{c i-v}=1 /(1+1)=50 \% \\
& \operatorname{tur}_{c-i-v}=1 /(1+1+1) \approx 33 \%
\end{aligned}
$$

For civ, its tur is $100 \%$, as there are no additional transactions to do the pre-deployment. For ci-v, and c-i-v methods, their turs are $50 \%$ and approximately $33 \%$ respectively, which indicates a relatively big waste from the user's view. Then if functions of a smart contract are called only few times, the civ method saves more transactions.

If we consider the termination step, the difference is bigger.

$$
\begin{aligned}
& \text { tur }_{\text {civt }}=1 / 1=100 \% \\
& \text { tur }_{c-i-v-t}=1 /(1+1+1+1)=25 \%
\end{aligned}
$$

While if the amount of functional invocations is big enough (e.g. infinite), the tur of ci-v methods is also close to $100 \%$, as shown (5). Although there is no big difference in this case, it still saves time for the first invocation, as it does not need to wait for the completion of the predeployment. At the same time, one transaction's fee is saved, it is meaningful if the project is a non-benefit project.

$$
\lim _{n \rightarrow \infty} t u r_{c i-v}=\lim _{n \rightarrow \infty} n /(n+1)=100 \%
$$

\subsection{Possibility of Dynamic Logic}

The civ method can also implement dynamical logic. The dynamical logic means that participants can delay their smart contracts to interact with the blockchain to the time when they are sending the functional transactions. When there is no requirement to pre-deploy a single smart contract for all participants, each participant can specify its smart contract at the sending time of functional transactions.

In this way, an application is composed of smart contracts from different participants. A smart contract can be changed if it has not been sent and no matter whether other smart contracts have been put to the blockchain or not. Note that previous work [5] proposed to split a single smart contract into several sub-contracts, aiming to facilitate the execution of a smart contract. In this paper, we use this method (together with the civ method) to implement the dynamical logic.

Here is an example of dynamic logic. It is a blockchain application, in which several participants exchange their assets. Each participant gives a smart contract to specify the conditions to exchange its assets. Matched smart contracts form an exchange. As we do not know who will join an exchange and their exchange condition previously, we adopt the dynamical logic. For example, a participant $(P A)$ specifies an exchange condition $(\operatorname{Con} A)$ that it gives 6 digital coins if it gets 5 assets; another one $(P B)$ has a plan to gives 5 assets if it gets 6 digital coins. As $C o n A$ and $C o n B$ matches, the exchange will be performed successfully. However, $P B$ wants to enhance its exchange condition from $C o n B$ to $C o n B^{\prime}$. Con $B^{\prime}$ requires the conditions of $C o n B$ plus a condition that $P B^{\prime}$ s balance is larger than 6 assets. Then when the asset balance of $P B$ is larger than 6 assets, the exchange is performed successfully. Thus, the application logic changes from $C o n A \& \& C o n B$ to $C o n A \& \& C o n B^{\prime}$.

In the above example, a participant specifies the condition only when it wants to exchange, which makes it difficult to set all possible exchange conditions previously. Then the civ method is used. The whole logic is the combination of smart contracts from all participants. Formally, we express the application logic (Logic) by logics from different participants $\left(e l_{n}\right)$, as in (6). $e l_{n}$ can be $C o n A, C o n B$ or $C o n B^{\prime}$, et. al.

$$
\text { Logic }=e l_{1} \& \& \ldots \& \& e l_{i} \& \& \ldots \& \& e l_{n}
$$

, where Logic is the logic of this exchange and $e l_{i}$ is the logic from participant $i$.

From (6), we can know that the whole logic is the intersection of logics of all smart contracts.

$$
\text { Logic } \subseteq \text { eli }
$$

Equation (7) indicates that when a new logic is added, agreements are narrowed down. Already existing logics are changed due to the immutability of the blockchain.

\subsection{Robust Analysis}

We try to analyze the robust when a transaction is missing. A missing transaction is a transaction that is sealed in the main chain first and later goes to a branch. It seems that this transaction is missing.

The missing probability is proportional to the number of transactions in the lifecycle. The reason is that each transaction is sealed separately and thus they are independent. Suppose there are $n$ transactions in a lifecycle, and then the probability for missing transactions $p_{\text {missing }}$ are as (8).

$$
p_{\text {missing }}=n * p
$$

, where $p$ is the probability that a transaction is missing.

A missing transaction may cause an error transaction, which is a transaction whose validation depends on a transaction while the dependent transaction is missing. An error transaction is one of the robust measurement of the blockchain system. The number of error transactions has a 
relationship to the number of transactions in the lifecycle. For simplification, we assume transactions in the lifecycle of a smart contract depends on its previous transaction. We arrange those transactions in the dependence order. Suppose the first missing transaction is at position $i$, and the number of transactions after this transaction is $n$. Thus, the number of all possible error transactions $\left(e n_{i}\right)$ is $n$, as all those transactions have a dependence on transaction $i$, as described in (9).

$$
e n_{i}=n
$$

For example, in the method of $\mathrm{c}-\mathrm{i}-\mathrm{v}$, transactions $\mathrm{v}$ and $\mathrm{i}$ depend on transaction c. If transaction c is missing, the number of error transactions are 2 (transaction $\mathrm{v}$ and transaction c). If transaction i is missing, the number of error transaction is 1 (transaction $\mathrm{v}$ ).

We further calculate the number of all possible error transaction cases (en). As each transaction has the possibility to be the first missing transaction, en is their summarization. Suppose there are $N$ transactions used in the lifecycle, then en can be expressed as (10).

$$
e n=\sum_{n=0}^{N-1} e n i=\left(N^{2}-N\right) / 2
$$

Formula (10) can help us to analyze the robust among different lifecycle methods. We first discuss the case when there is only one functional invocation $\left(n_{f u n}=1\right)$. In $\mathrm{c}-\mathrm{i}-\mathrm{v}$ method, $N$ is 3, and then $e n$ is 3 error transactions. In civ method, $N$ is 1 , en is 0 , which means there are no error transactions.

We further discuss two cases when the number of functional invocations is more than 1. (1) The function invocation time is $6\left(n_{\text {fun }}=6\right)$. For c-i-v, $N$ is 8 , which includes two additional lifecycle transactions, and en is 28. Similarly, en for civ is 15. (2) The functional invocations is relatively big $\left(n_{\text {fun }}=100\right)$. For c-i-v, $N$ is 102 , which includes two additional lifecycle transactions, and en is 5151. Similarly, $e n$ for civ is 4950 .

As en is high for $\mathrm{c}-\mathrm{i}-\mathrm{v}$ method, then the waiting time before the invocation should be long enough to ensure that the smart contract has been deployed correctly.

\subsection{Optimization Scope}

The smart contracts that can get more benefits from the optimization are mainly two types. The first one is the disposable smart contract, which changes frequently and is invoked few times. If the smart contract is deployed once and then its methods are invoked several times later, the optimization does not help much.

The second is the smart contract whose logic can be separated into different parts. It can form the dynamical logics, and provides the flexibility for each participant to delay its specific smart contract. If the smart contract must be a single file, it can only be delayed to the time when the first participants sending their request.

\section{Verification}

\subsection{Test Environment}

The blockchain in the verification is developed to support the lifecycle optimization model. There are three steps in this blockchain, putting the code of a smart contract to blockchain, instantiate the smart contract, and invocation of its function. Public available blockchains are not chosen as it is difficult to customize different lifecycle actions.

PoW is used as the consensus algorithm. The difficulty is set to the degree that the hash of a block starts with 6 zeros (it takes several seconds to mine a block in our verification environment). The mining reward is also giving to the block miner. If a miner mines a block successfully, it will get 50 internal coins. To get the rewards, miners on different nodes compete to find the nonce to match the required difficulty.

There is a smaller unit of internal coins, $l u$, as one internal coin is too big to use. This is similar to Ethereum, which has smaller units than one ether (its internal coin), such as wei ${ }^{1}$. The conversion from the internal coins to $l u$ is shown in (11).

$$
1 \text { internal coin }=10^{6} \mathrm{lu}
$$

We deploy 16 nodes in the verification blockchain. Those nodes are deployed in virtual machines of two workstations. Their computation resources are different, aiming to simulate different nodes configurations. The resources of memory are $1 \mathrm{G}, 2 \mathrm{G}, 4 \mathrm{G}$, or $8 \mathrm{G}$; the resources of CPUs are 1, 2, or 4 virtual CPUs $3.00(\mathrm{GHz})$. Those nodes form a P2P network, which is in the internal network of our lab.

\subsection{Comparison among Different Lifecycle Stage}

In this section, we compare different lifecycle methods. We compare their different processing time and different processing fees to reflect the different steps required in the lifecycle, and the transaction robust.

The smart contract for verification is a simple Java class with a length of 244 characters (base64 encoded). In the code putting stage, the smart contract is recovered by base 64 decoding. In the instantiation stage, the blockchain platform creates an instance of the target smart contract (a class instance in our verification). The invocation is to call the function that outputs the one message to the console. We choose only to output a message, because different smart contract languages may have different limitations for their callable functions, while all smart contract languages can output a message.

Totally, we have carried out 32 test rounds for the above four ways, civ, ci-v, c-iv, and c-i-v. Then we have 128 test rounds totally. In each round, steps $\mathrm{c}, \mathrm{i}$, and $\mathrm{v}$ are performed. It may be in a transaction (as in civ way), two transactions (as in c-iv or ci-v ways), or three transactions (as in c-i-v way). We use a script to judge and send the next transaction if the action of the previous transaction has been performed, aiming to eliminate the unnecessary waiting interval.

1. https://ethdocs.org/en/latest/ether.html 


\subsubsection{Processing Time}

In this section, we compare the processing time among different lifecycle methods. The processing time $\left(T_{\text {processing }}\right)$ is the period from the sending time of the first transaction to the time when the invocation is done. The processing time has two parts, the time to mining corresponding transaction into the blockchain $T_{\text {mining }}$, and the time taken by lifecycle actions $T_{\text {action, }}$ shown in (12).

$$
T_{\text {processing }}=T_{\text {mining }}+T_{\text {action }}
$$

We show the results of the processing time for c-i-v, ci-v, c-iv, and civ in Figure 4.

Fig. 4. The completion time of different lifecycle.

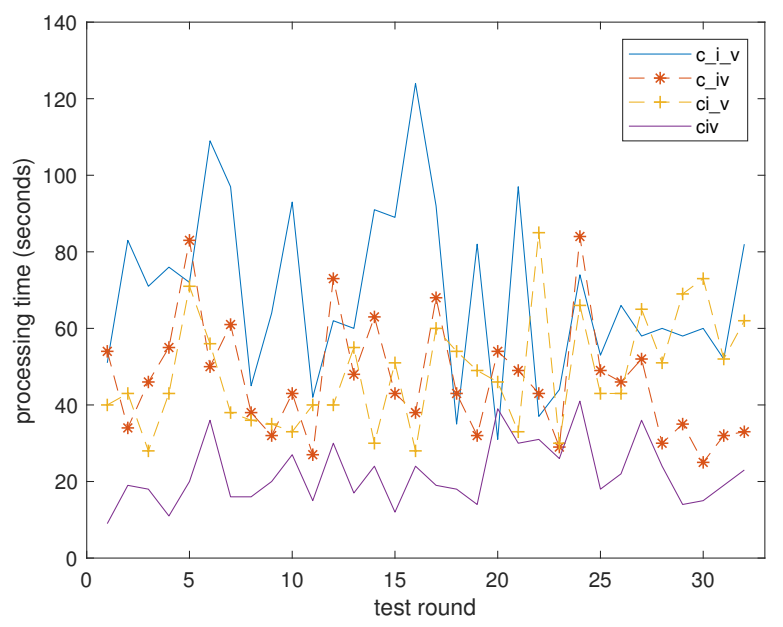

From Figure 4, we see that the processing time of the civ method is the lowest among those four methods, and its diagram is almost at the bottom. The minimum value of civ is 9 seconds at the first test round, and the maximum value is 41 seconds at the 24th test round. Methods of c-iv and ci-v are almost in the same tendency. The way of c-i-v has big peaks and change greatly. The processing time ranges from 31 seconds to 124 seconds. This indicates that the c-i$\mathrm{v}$ method is the most time-consuming method, and the civ method is the most time-saving method.

To give a clearer comparison, we show the average completion time, as in Figure 5. The average time of c-i-v is the longest. The processing time sequence is in (13).

$$
c-i-v>c i-v \approx c-i v>c i v
$$

The difference in the processing time indicates different numbers of transactions used in the lifecycle. There are two reasons. (1) $T_{\text {action }}$ has no big difference. Actions to process a smart contract are the same in different lifecycle methods, and then the actions processing time is almost the same. Then the difference is in $T_{\text {mining }}$. $(2) T_{\text {mining }}$ is longer than $T_{\text {action }}$. It takes several seconds to seal a transaction into the blockchain, while the time to process actions in step ' $c$ ', ' $i$ ', or ' $\mathrm{v}$ ' is less than 1 seconds on our test nodes. $T_{\text {mining }}$ is the major impact factor for the whole processing time.

In the civ way, there is only one transaction, and thus it only takes the least processing time. In the c-i-v way, there
Fig. 5. The average time of different lifecycle.

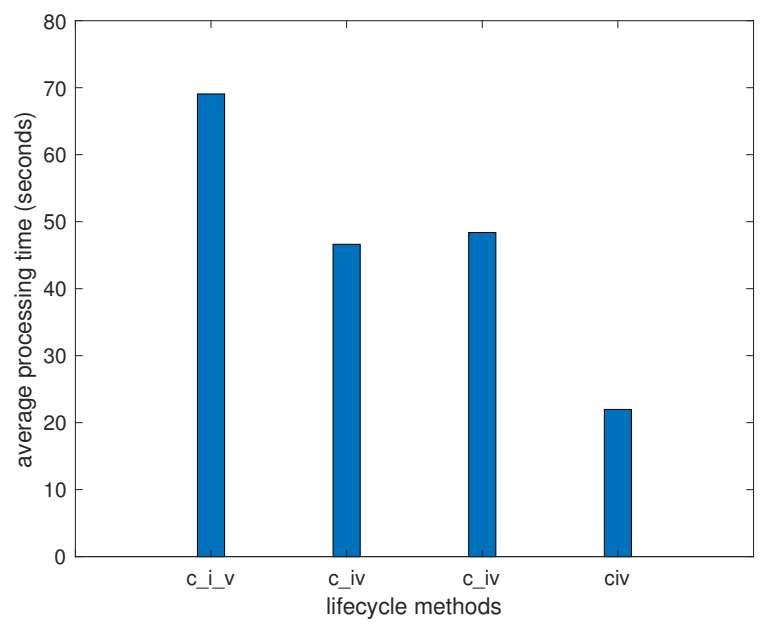

are three transactions, and it takes the longest time. There are two transactions for c-iv and ci-v, and their processing time does not have a big difference.

When the specific smart contract is only used once, the way of civ saves the package time of putting code and instantiation. This is one of the reasons why we propose the civ method.

\subsubsection{Processing fee}

In this section, we compare the processing fee among different lifecycle methods. As proposed in [6], it saves the transaction fee by the reduction of the number of used transactions.

The processing fee (processFee) is the rewards that the sender gives to the miner, which contains two parts, fixedFee, and smartContractFee, as in (14). fixedFee is the reward to package this transaction, which is a fixed value $(1000 l u)$ in our blockchain.

$$
\text { processFee }=\text { fixedFee }+ \text { smartContractFee }
$$

smartContractFee is the cost to run a smart contract, which is shown in (15). This fee is proportional to the line number of the source code file, fileSize. It should be proportional to the computational resources used for a smart contract, while for simplification we calculate this fee with respect to the file size. smartContractFee is also proportional to gasPrice, which is the reward for each line. The verification script changes gasprice with repect to on the burden of its sending node. If the CPU occupation on the sending node is high, gasPrice is high, aiming to control the transactions sending speed.

$$
\text { smartContractFee }=\text { gasPrice } * \text { fileSize }
$$

, where gasPrice is the cost to process one line of the source file and fileSize is the line number of the source file.

The result of the processing fee is shown in Figure6.

In Figure 6, the lowest processing fee is the way of civ, and the highest processing fee is the way of c-i-v. The other 
Fig. 6. The verification results of the processing fee, compared among four different methods (c-i-v, c-iv, ci-v, and civ).

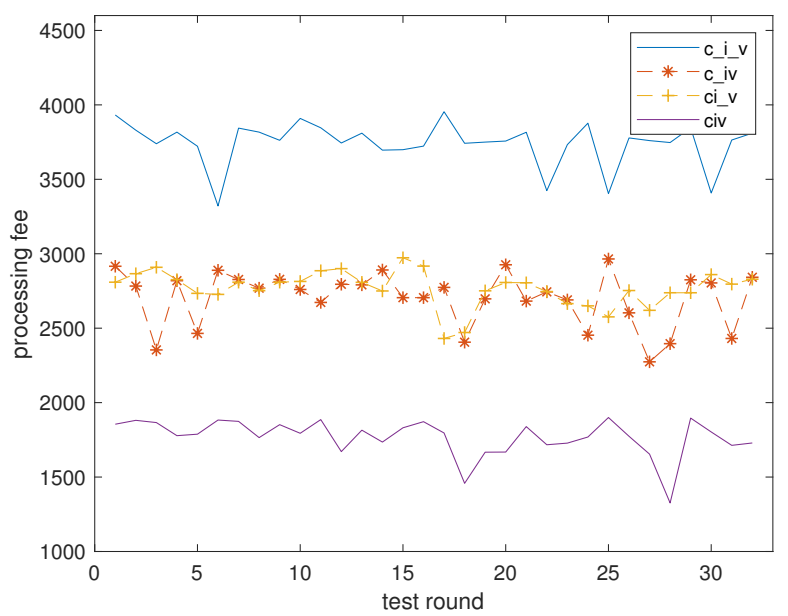

two ways are in the middle. The sequence of the processing fee is in (16).

$$
c-i-v>c i-v \approx c-i v>c i v
$$

This is also due to the different number of required transactions. In the $\mathrm{c}-\mathrm{i}-\mathrm{v}$ method, there are three transactions, and then it has three fixedFees. Its processing fee is more than $3000 \mathrm{lu}$. The minimum value is $3321 \mathrm{lu}$ at the 6th test round. Comparatively, there is only one transaction in the civ method, which makes its cost relatively low. Its highest processing fee is $1900 \mathrm{lu}$ at the 26th test round, far less than the minimum value of the $c-i-v$ method. The tendency of the processing fee is also reflected by the average processing fee. The average processing fees are $3743 l u, 2702 l u, 2766 l u$, $1768 l u$ for $\mathrm{c}-\mathrm{i}-\mathrm{v}, \mathrm{c}-\mathrm{iv}, \mathrm{ci}-\mathrm{v}$, civ respectively, which is shown in Figure 7.

Fig. 7. The processing fee used to process smart contracts for different lifecycle methods.

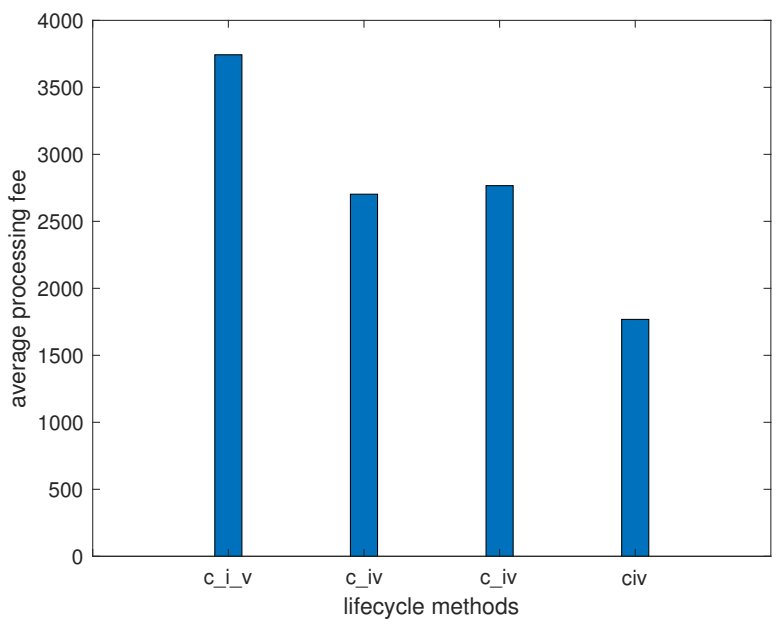

Although there is a noticeable difference in the whole process fee, there is no much difference in the fee required to process the smart contract (smartContractFee). The reason is that the processing smart files are the same and the actions required are also the same. To verify this, we subtract fixedFee from the above data; for c-i-v, $3000 \mathrm{lu}$ is subtracted as there are three transactions, $2000 \mathrm{lu}$ for ci$\mathrm{v}$ and $\mathrm{c}-\mathrm{iv}$ as there are two transactions, $1000 \mathrm{lu}$ for civ as there is one transaction. The result of smartContractFee is shown in Figure 8, and its average fees are shown in Figure 9.

Fig. 8. The gas fee required by smart contract (smartContractFee).

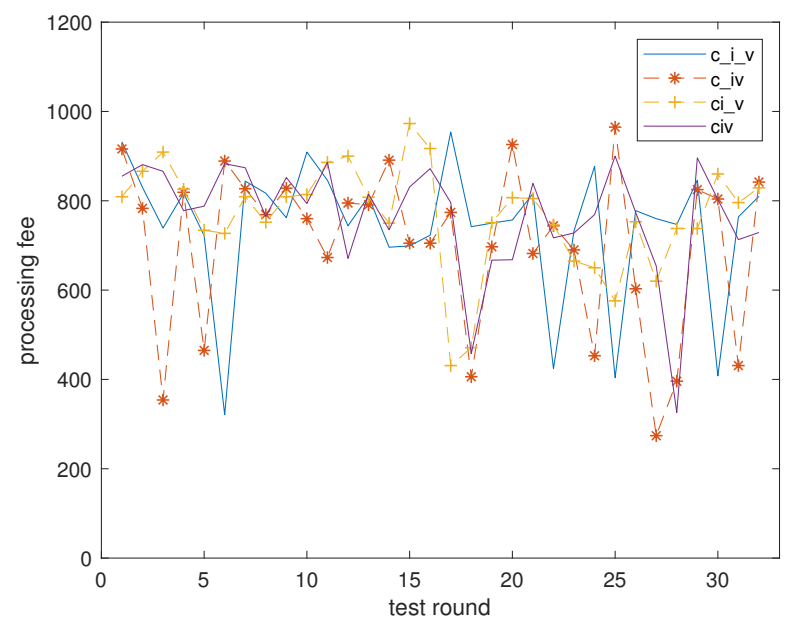

Fig. 9. The average gas fee required by the smart contract.

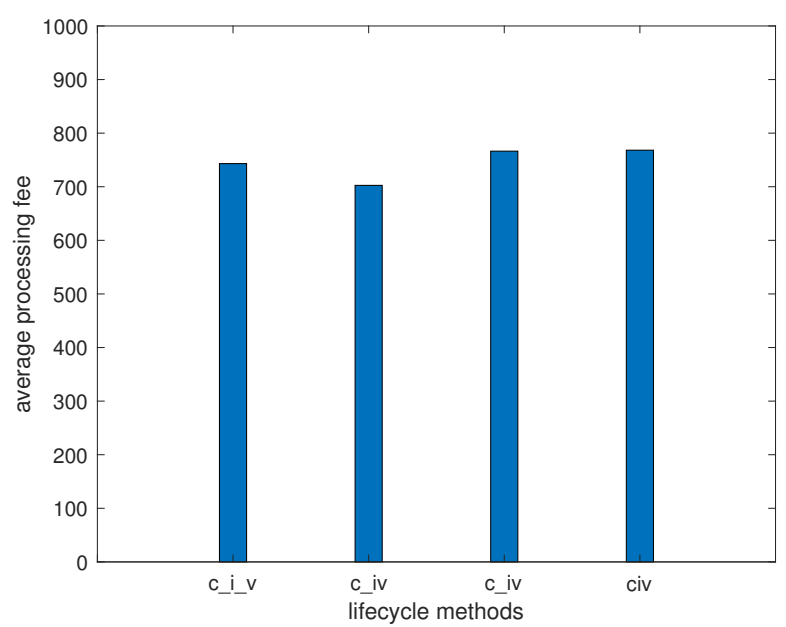

From Figure 9, we know that the fee used to process a smart contract is more than $700 l u$ and less than 770 $l u, 743.15 l u$ for c-i-v, $702.56 l u$ for c-iv, $766.40 l u$ for ci-v, and $768.21 \mathrm{lu}$ for civ. The biggest difference in the average processing fee is no more than $68 \mathrm{lu}$. The difference is due to the resource burden at different timestamps, as the gasPrice is higher in resource occupation is high and lower when the resource occupation is low.

In summary, the civ method saves money by the reduction of the fixed processing fees of additional transactions. 
The currently used method is similar to the ci-v way, which has more processing fees than civ due to an additional transaction. The method of c-i-v does not save any fixed processing fees.

\subsubsection{Transaction Robust}

We compare the robust among different lifecycle ways when some transactions are missing. Two aspects are analyzed, the missing transactions and the error transactions.

As it is difficult to control one transaction to be missing, this verification is a simulation instead of the verification on blockchains. We first set the probability of each transaction to be missing as 1/4 (use the rand function of Perl 5.14.0). Then we check the real missing transactions and the error transactions.

We carry out the test for 255 rounds. The results of the missing transactions are shown in Figure 10.

From the above, we see that the number of the missing transactions in the $\mathrm{c}-\mathrm{i}-\mathrm{v}$ way is the highest, 166 transactions, which is far more than 64 transactions $(1 / 4$ of 255 test rounds). The reason is that there are three transactions in one test round for the c-i-v method. Thus, it is about three times of 64 transactions. The method of civ has 69 missing transactions, which is about $1 / 4$ of 255 test round.

The results of error transactions are shown in Figure 11.

From the above results, we see that there are 143 error transactions for c-i-v method, 42 error transactions for ci$\mathrm{V}$, and 0 error transaction for civ method respectively. The error transaction of c-i-v is 3.4 times of ci-v method. The reason is that there are more possibilities for c-i-v method to have error transactions. First, if transaction $\mathrm{c}$ is missing, two transactions (for step $\mathrm{i}$ and $\mathrm{v}$ ) are error transantions. This happened at test round 9 and the other 47 test rounds. There is no such case (two error transactions when one transaction is missing) for the ci-v method. Second, the c-i-v has one more transaction (for step ' $i$ ') than the ci-v method. This transaction can also cause transaction $\mathrm{v}$ to be an error transaction.

There are no error transactions for the method of civ. The reason is that all Lifecycle steps are in a transaction. They are missing together or exists together. Thus, there is no chance to make only partial transactions exist.

The waiting time can also be saved from the roust point of view. As in methods of c-i-v and ci-v, the possibility of the missing and error transactions are high, it has to wait for several blocks to ensure that the previous transaction(s) will not be re-branched. While in the civ method, all operations are in one transaction, and there is no need to wait for the previous steps to complete.

\subsection{Flexible for Dynamical Logics}

In this section, we verify that the civ method is flexible for dynamical logics, which allows users to change their smart contracts to be sent even after other participants have given their smart contracts.

The verification scenario is an exchange between two users. User 1 wants to use its asset (asset1) to exchange the asset (asset2) of user 2. The exchange is originally under some conditions, referring to table 2 . However, during the exchange, user 2 wants to add an additional condition. This
TABLE 2

Original Exchange Scenario.

\begin{tabular}{clll}
\hline user & have & want & condition \\
\hline 1 & 50 asset1 & 20 asset2 & $\begin{array}{l}\text { pay 10 asset1 to user 2 when } \\
\text { getting 20 asset2 from user 2 } \\
\text { pay 20 asset2 to user 1 when } \\
\text { getting 10 asset1 from user 1 }\end{array}$ \\
\hline
\end{tabular}

requires changing the condition (user 2's smart contract) after the exchange begins. The civ method is flexible for this requirement, as no pre-deployed smart contract(s) are required. In this way, each user sends its condition in a civ transaction. Then, both user 1 and user 2 have the ability to change its condition of the exchange before sending its condition (by a civ transaction).

One corresponding verification has been carried out and the result is shown in Figure 12. First, user 1 sends its transaction, which includes a smart contract $(s c 1)$ to specify the exchange condition of user 1 . This transaction is sealed into blockchain at 4:08:42. Now the exchange contains the smart contract $s c 1$ as in (17).

$$
\text { Exchange }=s c 1
$$

The original exchange condition of user 2 is in another smart contract, $s c 2$. When the process goes on, user 2 wants to have more restrictions. Then it sends out a transaction with the new restriction of $s c 2^{\prime}$, which is sealed into the blockchain at 4:12:25. Now, this exchange is under two restrictions. All smart contracts match, and then the exchange completes, asset1 transferred to user 2, and assteType2 transferred to user 1 .

$$
\text { Exchange }=s c 1 \& \& s c 2^{\prime}
$$

From, this verification, we see that the exchange logic changes from $s c 1 \& \& s c 2$ to $s c 1 \& \& s c 2^{\prime}$. Meanwhile, the requirement (condition) of each user has been ensured. From (18), we see that the exchange contains the requirement of both user 1 and user2. When user 2 changes its exchange condition, user 1's condition still matches.

\section{Related Work}

Related works are mainly in three aspects: (1) to focus on the deployment, (2) to optimize the smart contract model, and (3) to save the gas fee.

The deployment-related issues are one research field. [7] proposes an off-chain executive module to save an additional smart contract to be deployed. [8] proposes to check the code correctness before deployment to avoid potential bugs, whose methods include bytecode analysis, source code analysis, and even dynamical code analysis. [9] propose a graphical method to deploy smart contract in a verified way, which helps to eliminate the errors during the deployment process.

There are several works to optimize the smart contract model. [5] proposes to divide a single smart contract into smaller sub-contracts (including a root contract together with molecular contracts and atomic contracts), which aims 
Fig. 10. Results of the missing transaction.
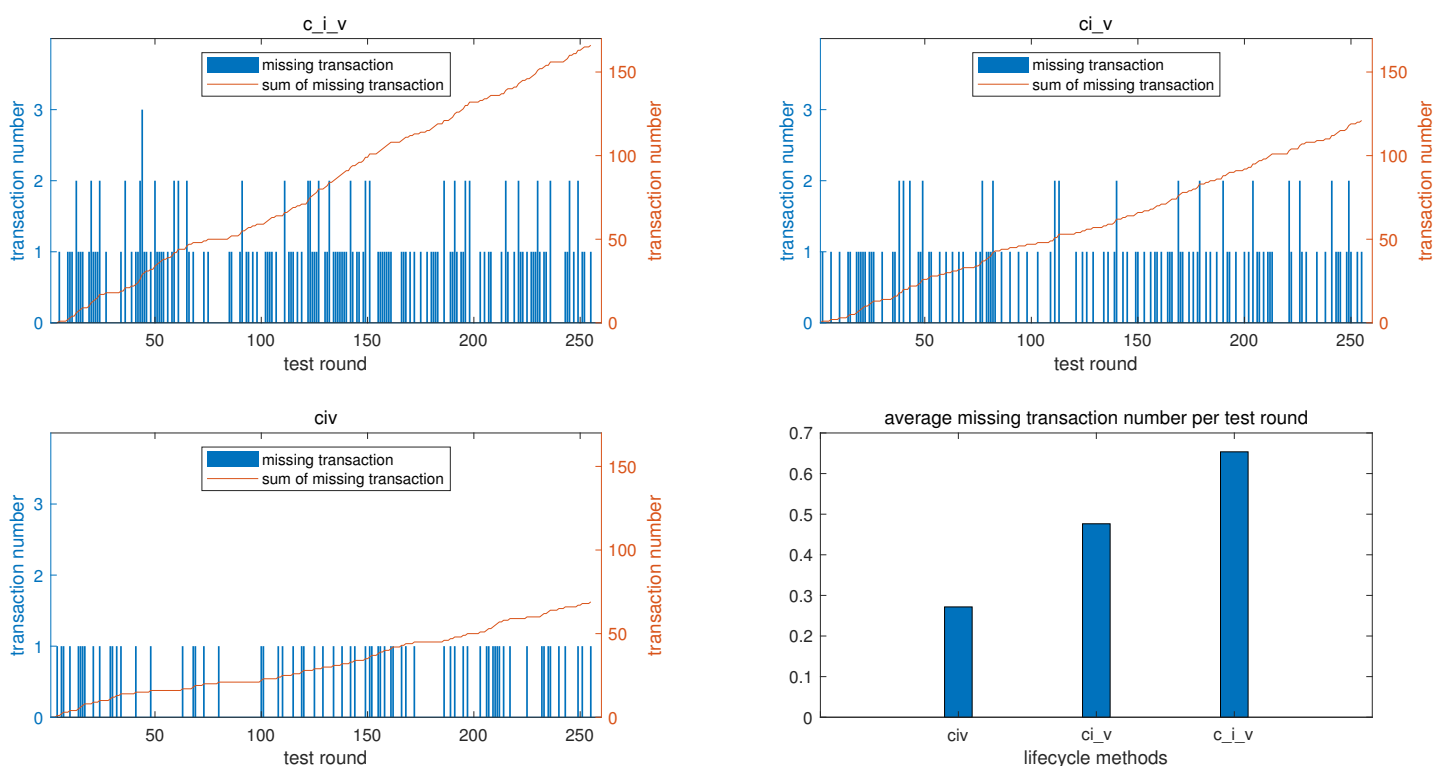

Fig. 11. Results of error transaction for C-i-v, ci-v, and civ. Note in the civ method, the error transaction number is 0 , and there is no figure in that figure.
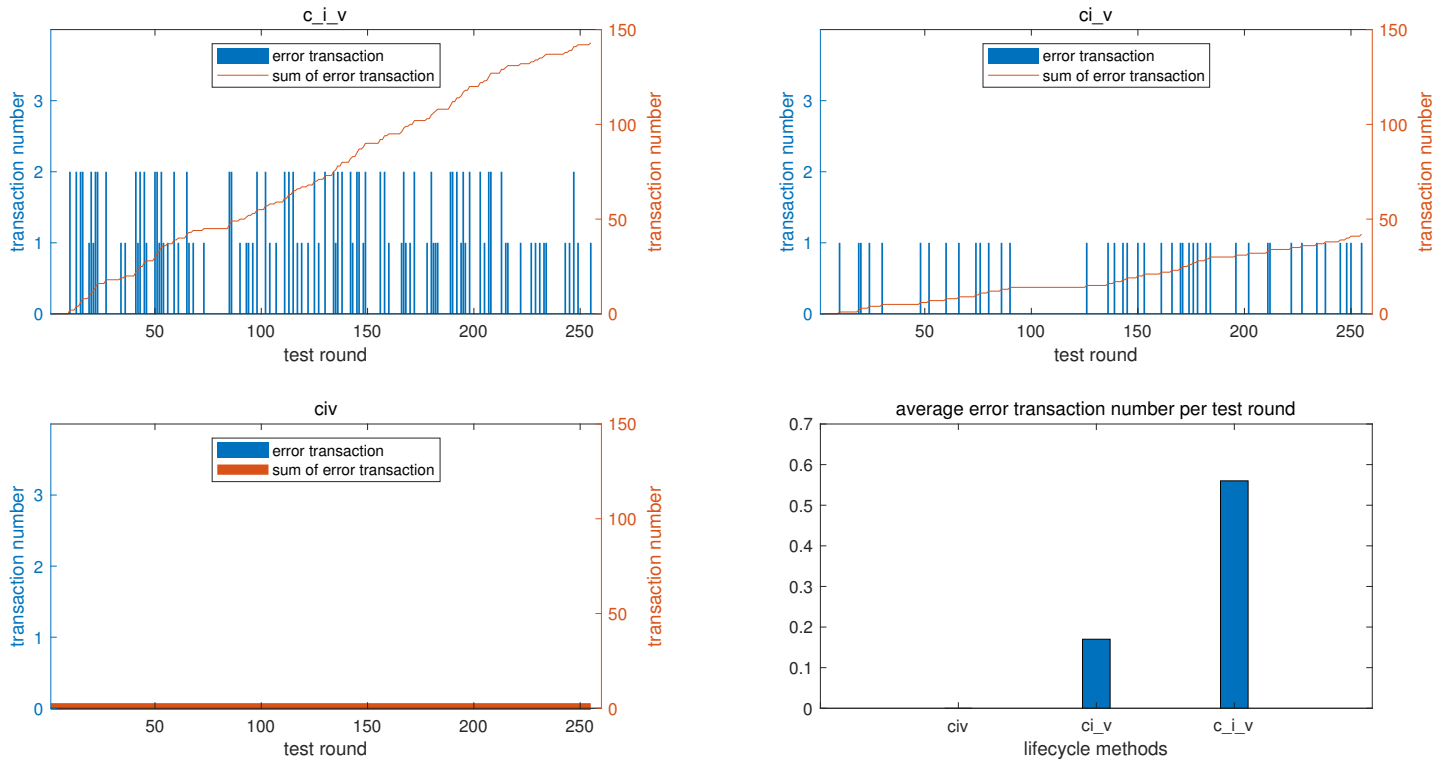

to facilitate the execution of smart contracts. [10] proposes to optimize business process smart contracts by extending the smart contract model to Petri nets. [11] proposes to optimize a smart contract at two aspects, (1) to save cost by optimization in the initialization and task execution steps, and (2) to increase maximized throughput by improved runtime components. Optimization of the smart contract is also done for special fields. For IoT, special issues include resource limits, bandwidth limits, connectivity limits, and et al. [12] proposes a blockchain-based IoT model to solve privacy and security threats for the resource-constraints of IoT networks.

Some works focus on saving the gas fee for the smart contract during the execution of the smart contract. One kind of work is to reduce the extra steps required by a smart contract. [7] proposes a cost-effective method by reducing extra smart contracts when interacting with data carriers. Another kind of work is to use design patterns. [14] points out that smart contracts with gas-inefficient code cost more than necessary. Then programming patterns are used in gas optimization, which identifies the patterns that cost additional gas fees and try to avoid those issues. [13] classifies several categories that may cause the wastes of gas, including external transactions, storage, memory, op- 
Fig. 12. Result of the exchange with an embedded smart contract.
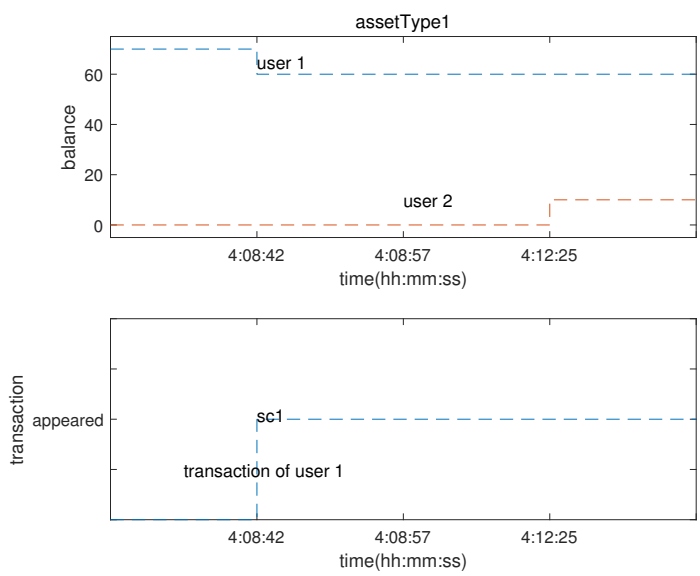

erations. Based on those patterns, it proposes solutions for corresponding problems.

\section{Conclusion}

In this paper, we address the pre-deployment issue of smart contracts, which is required for all smart contracts even if a smart contract is used only one time (disposable smart contracts). We proposed the model to combine the deployment steps and the first invocation step. This facilitates the usage of smart contracts by saving a separate pre-deployment step. The address of the disposable smart contract is even not required, as there are no invocations from other transactions. Meanwhile, this model saves an additional transaction, and thus it saves an additional transaction fee and the time to wait for the deployment transaction being put to the blockchain. Together with the smart contract separation, it allows the participants to delay their smart contracts to the request time (the dynamical logic). It makes the usage of smart contracts more flexible. The verification results show this model can save the gas fee, shorten the waiting time, increase the robust, and allow the dynamical logic. Overall, we provide a way to optimize the lifecycle of a smart contract.

\section{ACKNOWLEDGMENTS}

\section{REFERENCES}

[1] Mengelkamp E, Notheisen B, Beer C, et al. A blockchain-based smart grid: towards sustainable local energy markets[J]. Computer Science-Research and Development, 2018, 33(1-2): 207-214.

[2] Zhang B, Jiang C, Yu J L, et al. A contract game for direct energy trading in smart grid[J]. IEEE Transactions on Smart Grid, 2016, 9(4): 2873-2884.

[3] Liang W, Zhang D, Lei X, et al. Circuit Copyright Blockchain Blockchain-based Homomorphic Encryption for IP Circuit Protection[J]. IEEE Transactions on Emerging Topics in Computing, 2020.

[4] Sillaber C, Waltl B. Life cycle of smart contracts in blockchain ecosystems[J]. Datenschutz und Datensicherheit-DuD, 2017, 41(8): 497-500.

[5] Feng, T., Yu, X., Chai, Y. and Liu, Y. (2019), "Smart contract model for complex reality transaction", International Journal of Crowd Science, Vol. 3 No. 2, pp. 184-197.

[6] Hukkinen, Taneli. "Reducing blockchain transaction costs in a distributed energy market application." (2018).
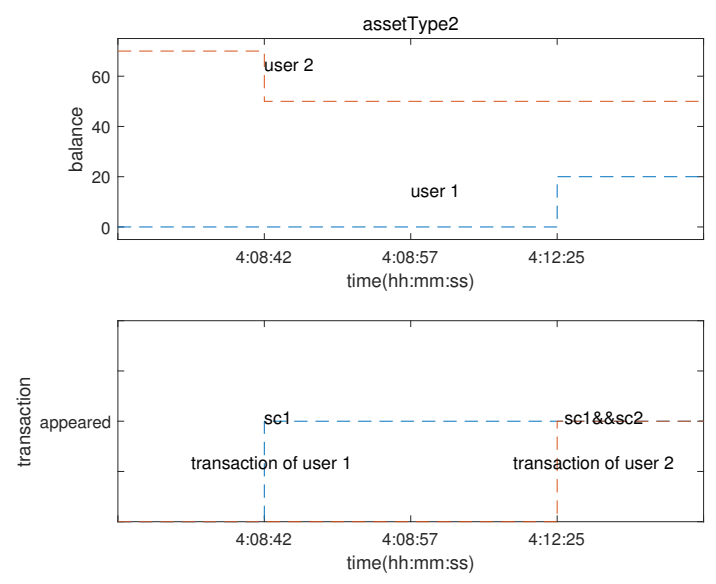

[7] Liu, Xiaolong, et al. "Elastic and cost-effective data carrier architecture for smart contract in blockchain." Future Generation Computer Systems 100 (2019): 590-599..pdf

[8] Zheng, Zibin, et al. "An overview on smart contracts: Challenges, advances and platforms." Future Generation Computer Systems 105 (2020): 475-491.

[9] Nelaturu, Keerthi, et al. "Verified development and deployment of multiple interacting smart contracts with VeriSolid." 2020 IEEE International Conference on Blockchain and Cryptocurrency (ICBC). IEEE, 2020.

[10] W. Hu, Z. Fan and Y. Gao, "Research on Smart Contract Optimization Method on Blockchain," in IT Professional, vol. 21, no. 5, pp. 33-38, 1 Sept.-Oct. 2019, doi: 10.1109/MITP.2019.2923604.

[11] García-Bañuelos L., Ponomarev A., Dumas M., Weber I. (2017) Optimized Execution of Business Processes on Blockchain. In: Carmona J., Engels G., Kumar A. (eds) Business Process Management. BPM 2017. Lecture Notes in Computer Science, vol 10445. Springer, Cham.

[12] A. D. Dwivedi, L. Malina, P. Dzurenda and G. Srivastava, "Optimized Blockchain Model for Internet of Things based Healthcare Applications," 2019 42nd International Conference on Telecommunications and Signal Processing (TSP), Budapest, Hungary, 2019, pp. 135-139, doi: 10.1109/TSP.2019.8769060.

[13] L. Marchesi, M. Marchesi, G. Destefanis, G. Barabino and D Tigano, "Design Patterns for Gas Optimization in Ethereum," 2020 IEEE International Workshop on Blockchain Oriented Software Engineering (IWBOSE), London, ON, Canada, 2020, pp. 9-15, doi: 10.1109/IWBOSE50093.2020.9050163.

[14] Chen T, Feng Y, Li Z, et al. GasChecker: Scalable Analysis for Discovering Gas-Inefficient Smart Contracts[J]. IEEE Transactions on Emerging Topics in Computing, 2020.

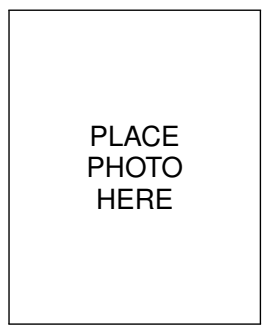

Hong Su is a Ph.D. candidate in the College of Computer Science, Sichuan University. He received the M.S. degree from Sichuan University of China in 2006. His current research interests include blockchain and Value Internet. Contact him at suguest@126.com. 